\title{
Cloud based RC Book Verification
}

\author{
M. Muthugayathridevi, M. Archana, P. Poothapandhichi, N. Arumugam
}

\begin{abstract}
In the field of transportation, there are such a large amount of documents want to be maintained for every automobile.So, at the same time as driving on public roadways each car proprietor has to carry all of the updated documents of a automobile with them. But it is very hard for proprietor to preserve all of the car documents like RC e book, insurance details, and greater collectively with them always. The misplacement or damaging of files due to climatic situations or as time passes are the biggest issues. If the driving force forgot to carry every body of these documents, he want to present heavy fines. With this "Cloud Based RC book Verification using RF transmitter and Receiver", vehicle documents can be electronically verified which helps in time consumption. The receiver identifies each car with unique frequency emitted from character transmitter. The $R F$ receiver is connected to a cloud, this cloud shops all of the documents related to automobile and fetches all the information associated with the automobile, the statistics's includes RC ebook, proprietor details, smoke check details, and more. This idea may also be helps to tune stolen with the assist of authorities.
\end{abstract}

Index Terms: Rf Transmitter And Reciever,Node Mcu,Cloud Database.

\section{INTRODUCTION}

The technology has grown in recent years in many fields like communication, medical, transportation etc. All the devices that we are using is wise making our life easier and faster. All other fields, similarily the transportation field has also advanced and one in every of the most examples is that the GPS Systems in vehicles and control systems.As per the transport policies of each vehicle should should maintain some documents like RC Book, Pollution instrument panel certificate. And the files should be carried by proprietary always with the vehicle on public roadways. But it's very difficult for someone to hold the vehicle documents like RC book, insurance details, together with them always. If they forgot anyone of the documents during the police checking,

Revised Manuscript Received on April 04, 2020.

* Correspondence Author

M. Muthugayathridevi*, Electronics and Communication Engineering, National Engineering college,Kovilpatti,India. Email: srimgayathridevi@gmail.com.

M.Archana, Electronics and Communication Engineering, National Engineering college,Kovilpatti,India. Email: archanam199898@gmail.com

P.Poothapandhichi, Electronics and Communication Engineering, National Engineering college,Kovilpatti,India. Email: petchisasi1@gmail.com

N.Arumugam, Associate professror in Department of ECE, National Engineering College,Kovilpatti,India. Email:armsece@gmail.com.

(C) The Authors. Published by Blue Eyes Intelligence Engineering and Sciences Publication (BEIESP). This is an open access article under the CC BY-NC-ND license (http://creativecommons.org/licenses/by-nc-nd/4.0/) it'll result in paying unneccesary fines. If they forgot anyone of the documents during the police checking, it'll result in paying unneccesary fines. The proposed system "Cloud Based RC book Verification using RF transmitter and Receiver" helps both inspection team and vehicle owners. All vehicle documents are accessible using cloud server through RF Transmitter and Receiver. The receiver is connected to the Cloud, and fetches all the data about the vehicle like RC book, owner details, smoke test details, and more. The inspection team can easily understand and verifying all relevant information of both vehicle and its owner.

\section{RELATED WORK}

[1] Creates an answer for manual toll collection in transportation. An RF module at the toll booth reads the tag of the vehicle from a specific distance. The system authenticates the vehicle and deduct the mandatory fee from the owner's account and opens the toll gate upon a successful processing. [2] Discusses privacy and security protection of RF data in E-passport. E-Passport may be a machine-readable passport, which is biometrically enabled and globally interoperable.

[3] In a tracking system is developed to tracethe vehicle on public roadways. But it's very difficult for someone to hold the vehicle documents like RC book, insurance details, together with them always.

[4] Discusses a vehicle plate operated as a tag antenna since it's a conductor (Made of Aluminum).The reader antenna is mounted on a cantilever bracket on each roadway. Thus the reader can read the tag ID although the vehicle is way from the reader.

\section{CLOUD BASED RC BOOK VERIFICATION SYSTEM USING RF TRANSMITTER AND RECEIVER}

In generally, the wireless structures designer has to consider two basic constraints: it should function over a selected distance and transfer a specific quantity of information inside a know-how rate. These RF modules met these constraints. The RF modules are generally $433 \mathrm{MHz}$. The transmitter draws no strength while logic one is despatched service is fully on to about $4.5 \mathrm{~mA}$ with a 3volts electricity supply.F rom the RF transmitter the record is sent, which is obtained with the aid of the tuned receiver.For data transfer, RF transmitter and the receiver are interfaced with MCU.As RFID covers best small range of area we can use RF transmitting common sense 0 while completely suppressing the provider frequency hence consume notably low strength in battery operation. When good judgment one is shipped service is absolutely directly to about $4.5 \mathrm{~mA}$ with a 3volts electricity supply.

Blue Eyes Intelligence Engineering \& Sciences Publication 
Transmitter and as a result the receiver are duly interfaced to 2 microcontrollers for statistics transfer.As RFID covers best small range of place we are visiting use RF Transmitter and Receiver This technique helps in covering larger area than RFID. In many projects we use RF modules for transmit and get hold of the statistics due to the fact it's excessive quantity of programs than IR. RF indicators travel inside the transmitter and receiver even while there's an obstruction.

RF transmitter ought to be constant in vehicle passing via the toll gate. RF receiver will are living at the toll gate .Each transmitter which is constant in vehicl will emit radio waves of various frequencies. If receiver is of bit then four exclusive form of frequency are travelling be acquired by the RF receiver . in the identical manner if RF receiver is of 4 bit then sixteen exclusive frequencies are traveling be received by RF receiver. Initially every database of person members have to be stored in cloud database via the server. Whenever a car passes thru the toll gate , RF transmitter transmits RF sign which is received with the aid of the RF receiver constant on the toll gate. Once RF signal is received it compares with cloud database and verifies whether the records base are accurate and valid.If all the informations are legitimate then toll gate opens and the automobile is allowed to move further.And also it is similar to Vehicle Documents Verification System using Advanced Digi-locker System [7].

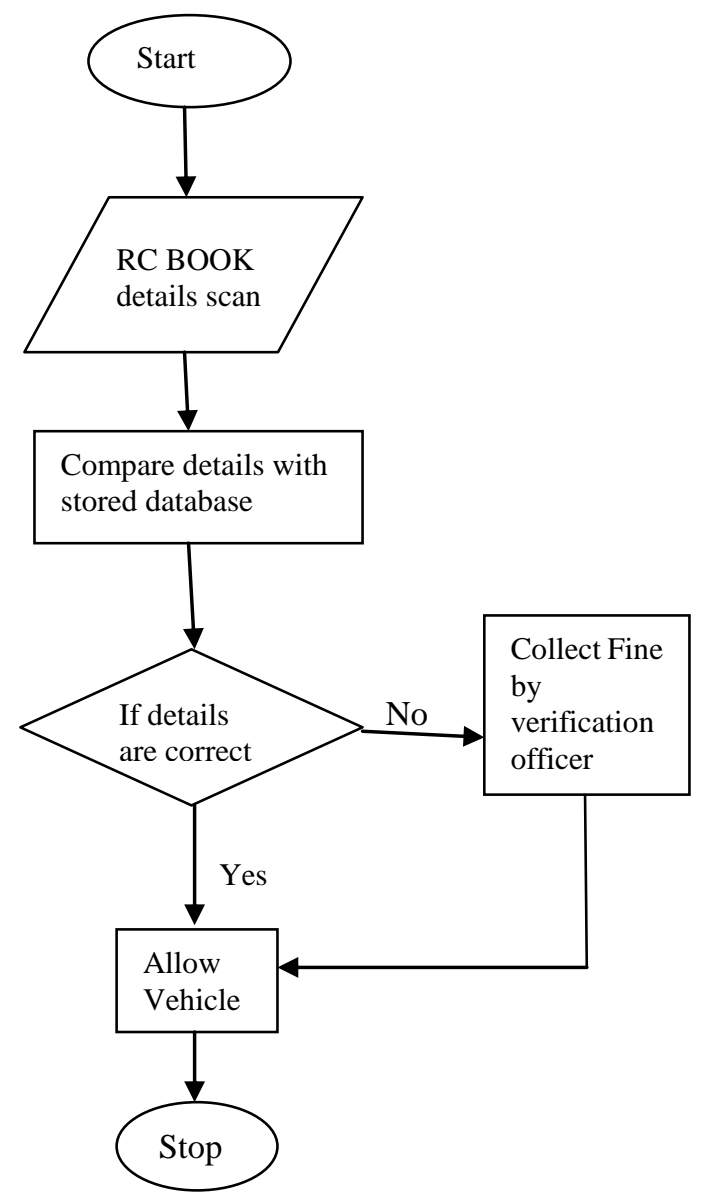

Fig.1.flow chart

\section{IMPLEMENTATION}

\section{A. Rf Modules}

RF modules are most often utilized in medium and low volume products for consumer applications likIn generally, the wireless structures designer has two overriding constraints: it should function over a particular distance and transfer a particular quantity of knowledge inside a know-how rate. These RF modules met this constraints. And the RF modules are $433 \mathrm{MHz}$. The transmitter draws no strength while logic one is despatched service is fully on to about $4.5 \mathrm{~mA}$ with a 3volts electricity supply. The records is distributed serially from the transmitter which is obtained with the help of the tuned receiver. Transmitter and also the receiver are duly interfaced to 2 microcontrollers for data transfer.As RFID covers best small range of area we will use RF transmitting sense 0 while completely suppressing the provider frequency hence consume notably low strength in battery operation. When wisdom one is shipped service is totally on to about $4.5 \mathrm{~mA}$ with a 3volts electricity supply. the info is distributed serially from the transmitter which is obtained by using the tuned receiver. Transmitter and as a result the receiver are duly interfaced to 2 microcontrollers for statistics transfer.As RFID covers best small range of place we are visiting use RF Transmitter and Receiver .It covers large area than RFID. In many projects we use RF modules for transmit and obtain hold of the statistics thanks to the very fact it's excessive quantity of programs than IR. RF indicators travel inside the transmitter and receiver even while there's an obstruction.RF transmitter must be constant in vehicle passing via the toll gate. RF receiver will live at the toll gate .Each transmitter which is constant in vehicle will emit radio waves of varied frequencies. If receiver is of bit then four exclusive type of frequency are travelling be acquired by the $\mathrm{RF}$ receiver . within the identical manner if RF receiver is of 4 bit then sixteen exclusive frequencies are traveling be received by RF receiver. Initially every database of person members need to be stored in cloud database via the server .Whenever a car passes thru the toll gate , RF transmitter transmits RF sign which is received with the help of the RF receiver constant on the toll gate. Once RF signal is received it compares with cloud database and verifies whether the records base are accurate and valid.If all the informations are legitimate then toll gate opens and also the automobile is allowed to maneuver further. They're sometimes accustomed replace older infrared communication designs as they have the advantage of not requiring line-of-sight operation Several carrier frequencies are commonly utilized in commercially available RF modules, including those within the commercial, scientific and medical (ISM)radio bands like $433.92 \mathrm{MHz}$, $915 \mathrm{MHz}$, and $2400 \mathrm{MHz}$. These frequencies are used thanks to national and international regulations governing the used of radio for communication. Short Range Devices may use frequencies available for unlicensed like $315 \mathrm{MHz}$ and 868 MHz. RF modules may befits an outlined protocol for RF communications like Zigbee, Bluetooth Low Energy, or Wi-Fi, or they'll implement a proprietary protocol . 4.2 RF TRANSMITTER An RF transmitter module could even be a little PCB sub-assembly capable of transmitting a radiation and modulating that wave to hold data.

\section{B. Rf Receiver}

The modulated RF signal is received by RF receiver module and it is demodulated. Super heterodyne receivers and super regenerative receivers are two kinds of RF receiver modules.

Published By:

Blue Eyes Intelligence Engineering

\& Sciences Publication

(C) Copvriaht: All riahts reserved.

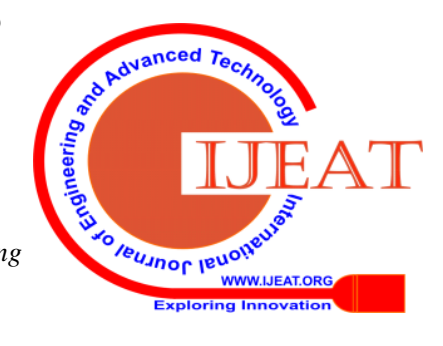


By employing amplifiers in series combination to extract modulated data from a carrier their cost is usually low and their power designs are low by. Considerably with temperature and power supply voltage their frequency of operation varies so, these modules are generally imprecise. Performance of Superheterodyne receivers are better than superregenerative; so,they provide increased accuracy. the soundness of Superheterodyne transceivers comes from a dearer fixed crystal design.Thus, advances in receiver chip design and fabrication results in little price difference between the superheterodyne and superregenerative receiver modules. An open-source firmware NodeMcu(Microcontroller unit) is development kit which helps in prototyping IoT product by means of script lines.It supported ESP8266 which is an Wifi Module,Which integrates GPIO(General purpose input outpu), PWM(Pulse width modula tion), IIC(serial two wire bus),and serial communication protocols.

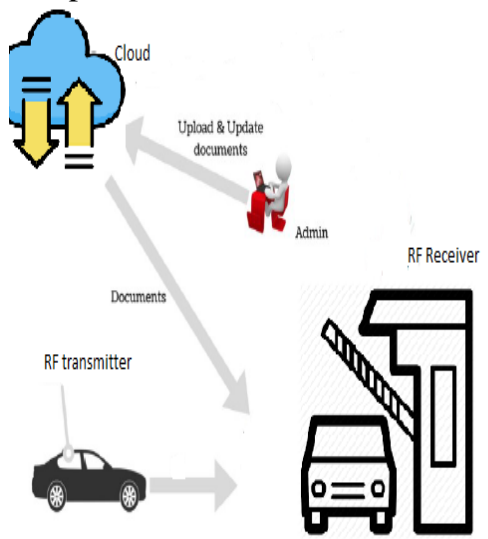

Fig.2.Implementation View

\section{Node Mcu}

Open-source firmware NodeMcu(Microcontroller unit) is development kit which helps in prototyping IoT product by means of script lines.Node MCU has ESP8266 which is an Wifi Module, Which integrates GPIO(General purpose input outpu), PWM(Pulse width modula tion), IIC(serial two wire bus), 1-Wire and ADC all in one board.

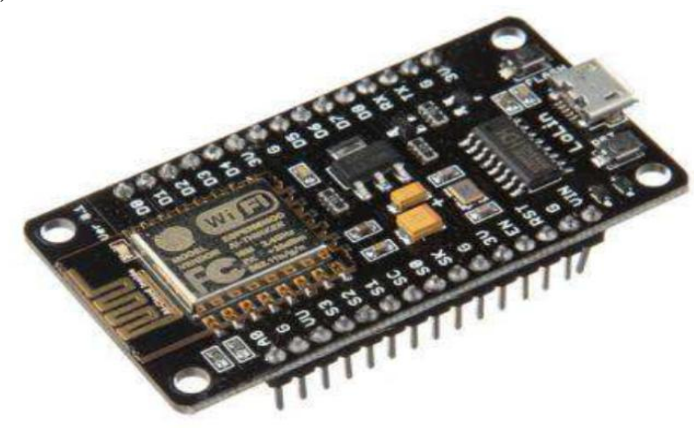

Fig.3. NODE MCU

Features

- Uses CH340G rather than CP2102.

-Superior stability on all supported platforms, NodeMCU has integrated USB-TTL serial with awesome dependable industrialstrengthCH340G.

- Wireless $802.11 \mathrm{~b} / \mathrm{g} / \mathrm{n}$ standard

- WiFi at 2.4GHz, guide WPA / WPA2 safety mode

- Support STA/AP/STA + AP 3 operating modes

- Built-in internet protocol TCP/IP stack to support multiple TCP Client connections (5 MAX).

\section{Software Used}

PHP springs from Personal Home Page Tools and started out out as alittle open source challenge that developed as an increasing number of people revealed how beneficial it really was. Back in 1994 Rasmus Lerdorf unleashed the number one model of PHP.This server side scripting language is employed in many packages such the manner to control dynamic content, databases, session tracking, even construct complete e-trade sites. This scripting language has also help for several of the net servers today.Some of the commonest net servers supported by means of PHP are Caudium, Xitami, OmniHTTPd.

\section{E. My Sql}

MySQL is additionally a fast,user friendly Relational database management systems used being employed for several small and massive businesses. MySQL is becoming so popular and it is an powerful program in its claim. MySQL can works on many running systems and PERL,PHP, C, C++, JAVA etc languages.
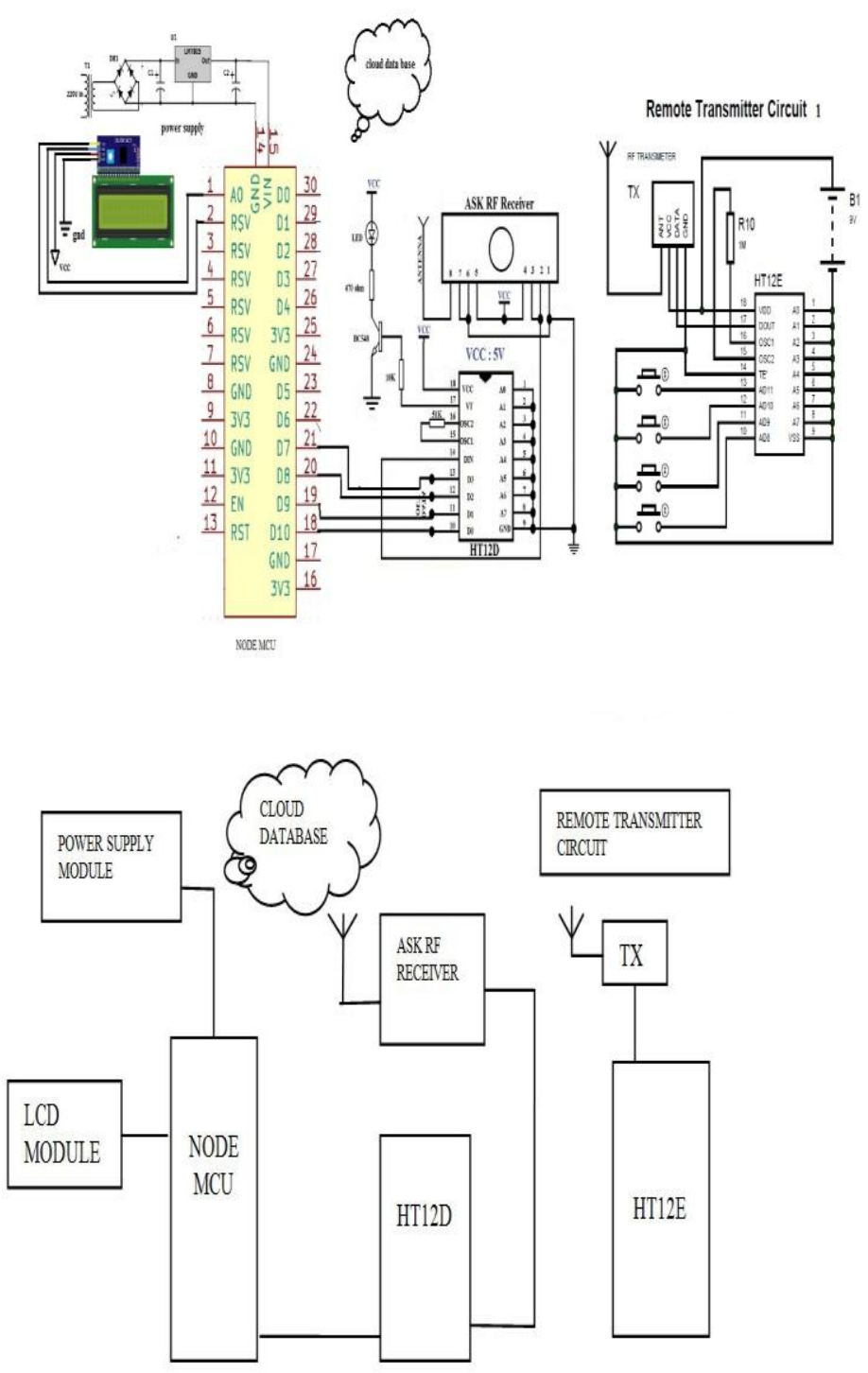

Fig.4. Block Diagram

Published By:

Blue Eyes Intelligence Engineering \& Sciences Publication

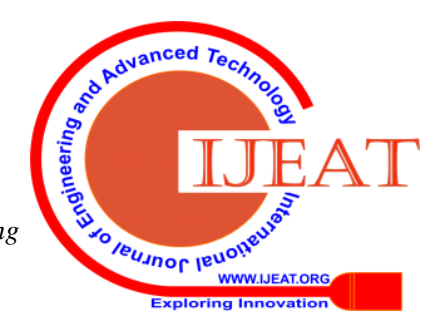




\section{OUTPUT}

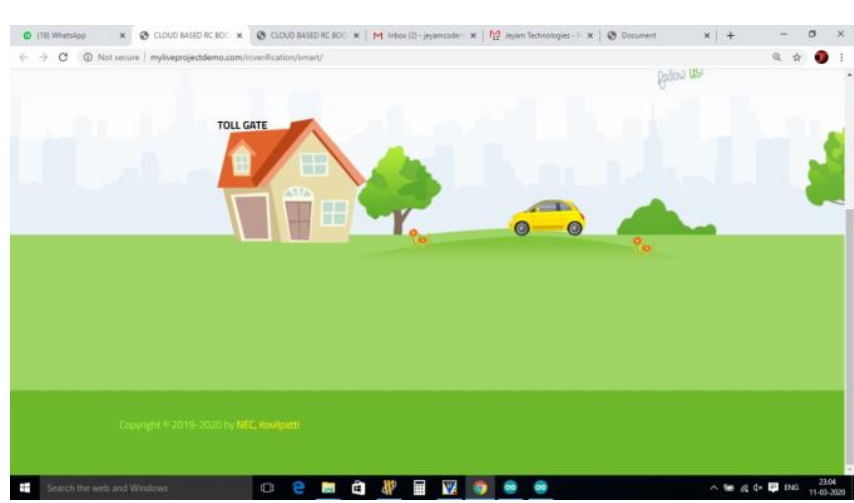

Fig.5. Output When The Vehicle Is Enter To The Tollgate

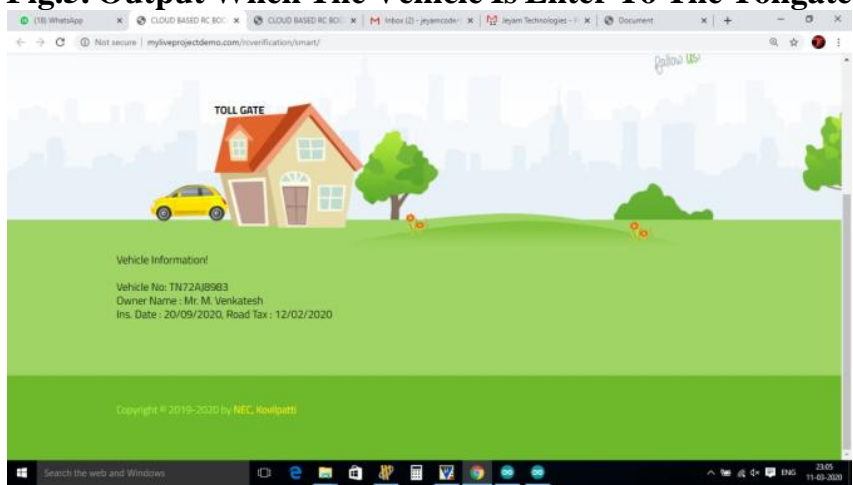

Fig.6.Output When The Vehicle Crosses The Tollgate

In the figure 5 the vehicle is enter to the tollgate the receiver set in the tollgate still waiting for the signal to receive from the vehicle.

In the figure 6 car is crossing the toll gate and receiver starts receiving signal from the transmitter fixed in the vehicle.After the successful verification of RC book the vehicle is allowed to pass through the tollgate otherwise the gate not be opened.

\section{RESULTS AND CONCLUSION}

Our system has been tested by fixing RF module on the automobile. The reader successfully identifies the ID from the remote transmitter and loads the scaned documents from the database. The documents details has been displayed on the display unit and therefore the verifying officer at the receiver end verifies the RC book information which has been already updated within the database. All the vehicle's documents is accessed electronically and this method creates an answer for manual RC book checking in transportation field and helps in time consumption for both the inspection team and vehicle owners.

\section{REFERENCES}

1. Pinaki Ghosh, Dr. Mahesh T R " A Privacy-Preserving Mutual Authentication Protocol for RFID based Automated Toll Collection "ICT in Business Industry\& Government (ICTBIG), International Conference on 18-19 Nov. 2016

2. Md, Monzur Morshed, "Privacy and Security Protection of RFID Data in E-Passport", Software, Knowledge Information, technology and Applications (SKIMA), 2011 5th International Conference on 8-11 Sept. 2011

3. Daniel Iturralde, Nestor Becerra "A new system supported web services and RFID for tracking people in a very pervasive mining environment" Communications (LATINCOM), 2013 IEEE Latin-America Conference on 24-26 Nov. 2013

4. Zhipeng Liang, "Design of vehicle plate RFID Tag Antenna Using Characteristic Mode Pattern Synthesis", IEEE Transactions on Antennas and Propagation ( Volume: 65, Issue: 10, Oct. 2017 ) 4964 -4970 .

Published By:

Blue Eyes Intelligence Engineering \& Sciences Publication

N.Arumugam Specialized in vlsi and sensor networks and he is associate professor at National Engineering college,Kovilpatti,India and has 32 years teaching experience.Have done many funded projects and researches in various areas.

Mrchana pursuing her Batchelor of Engineering in the domain of Electronics and Communication Engineering at completed many online courses. Her area of interests are Internet of Things and Cloud computing,Circuit Analysis,Medical Electronics.

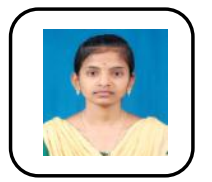

P.Poothapandhichi pursuing her Batchelor of Communication Engineering at National Engineering college,Kovilpatti,India.She attended many technical workshops and participated in many events conducted by different college.Her area of interests are Wireless communication,Network Theory,Radar.She was a active member of IEEE.

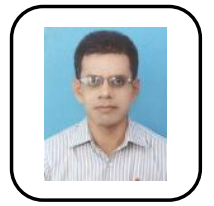

